\title{
The Rotation Set of a Homeomorphism of the Annulus is Closed
}

\author{
Michael Handel
}

Department of Mathematics and Computer Science, Lehman College, Bronx, NY 10468, USA

\begin{abstract}
We show that the rotation set for any orientation preserving, boundary component preserving homeomorphism of the annulus is closed. In particular, if the homeomorphism is area preserving, then the rotation set is a closed interval.
\end{abstract}

\section{Introduction}

In this paper we show that for any orientation preserving, boundary component preserving homeomorphism $f: \mathbf{A} \rightarrow \mathbf{A}$ of the closed annulus, and for any lift $\widetilde{f}: \tilde{\mathbf{A}} \rightarrow \tilde{\mathbf{A}}$ to the universal cover, the rotation set $R(\widetilde{f})$ is closed. This generalizes work of Aubry [Au-D] and of Mather [Ma] who showed (among other things) that if $f$ is an area preserving twist map of the annulus, then $R(\tilde{f})$ is a closed interval.

We identify $\tilde{\mathbf{A}}$ with $\mathbf{R} \times[0,1]$ and let $p_{1}: \tilde{\mathbf{A}} \rightarrow \mathbf{R}$ be the projection onto the first coordinate. The covering translation for $\tilde{\mathbf{A}}$ is $T(x, y)=(x+1, y)$. For each $x \in \mathbf{A}$, choose a lift $\tilde{x} \in \tilde{\mathbf{A}}$ and consider $\lim _{n \rightarrow \infty}\left(p_{1} \tilde{f}^{n}(\tilde{x})-p_{1}(\tilde{x})\right) / n$. When this limit exists, it is called the $\tilde{f}$-rotation number of $x$ and denoted $\varrho(\tilde{f}, x)$; as the notation indicates, it is independent of the choice of $\tilde{x}$. Although $\varrho(\tilde{f}, x)$ need not be defined for all $x$, it is defined $\mu$-a.e. for every $f$-invariant measure $\mu$.

Theorem 0.1. If $f: \mathbf{A} \rightarrow \mathbf{A}$ is an orientation preserving, boundary component preserving homeomorphism and $\tilde{f}: \tilde{\mathbf{A}} \rightarrow \tilde{\mathbf{A}}$ is any lift, then:

1. The rotation set $R(\tilde{f})=\bigcup \varrho(\tilde{f}, x)$ is a closed set, where the union is taken over the domain of $\varrho$.

2. For each $r \in R(f)$, there is an $f$-invariant measure $\mu_{r}$ such that $\varrho(f, x)=r$ for $\mu_{r}$-a.e. $x \in \mathbf{A}$.

3. With the exception of at most a discrete set of values $r$ in $R(\tilde{f})$, there is a compact invariant set $Q_{r}$ such that $\varrho(\tilde{f}, x)=r$ for all $x \in Q_{r}$; if $r$ is rational then $Q_{r}$ exists and is realized by a periodic orbit. 
Remark. I do not know any examples for which there exists $r \in R(\widetilde{f})$ with no compact invariant set $Q$ satisfying $\varrho(\widetilde{f}, x)=r$ for all $x \in Q_{r}$.

Remark. For each $x \in \mathbf{A}$, let $\varrho^{\prime}(\widetilde{f}, x)$ be the set of accumulation points of $\left\{\left(p_{1} \tilde{f}^{n}(\tilde{x})\right.\right.$ $\left.\left.-p_{1}(\tilde{x})\right) / n: n \geqq 1\right\}$ and let $R^{\prime}(\tilde{f})=\bigcup_{x \in \mathbf{A}} \varrho^{\prime}(\tilde{f}, x)$. Our proof of Theorem 0.1 extends in a straightforward way to show that $R^{\prime}(\tilde{f})=R(\tilde{f})$.

The proof of Theorem 0.1 can be divided into three parts. Although there are no pseudo-Anosov homeomorphisms on an annulus, there are homeomorphisms that are pseudo-Anosov relative to a finite invariant set. The first step in the proof (Proposition 1.1) is to show that for this special class of homeomorphisms, the rotation set is a closed interval and that each point in this interval is realized on a compact invariant set. The proof is a straightforward application of symbolic dynamics.

The next step is to exploit the fact that a pseudo-Anosov homeomorphism has the minimal complexity among all homeomorphisms in its homotopy class. We show (Proposition 1.2) that if $\phi$ is pseudo-Anosov relative to $K$, and if $f$ is homotopic to $\phi$ relative to $K$, then the interior of the rotation set for $\phi$ is contained in the rotation set for $f$ and that every element of this open interval is realized on an $f$-invariant compact set. The proof is a simple extension of the global shadowing techniques in [H2].

The third part of the proof is to show that if certain limit arguments (taking Hausdorf limits of $\omega$-limit sets) do not succeed in producing an invariant set with rotation number $r$, then the pseudo-Anosov techniques of steps 1 and 2 are applicable. This step is contained in Sects. 2 and 3. The limiting arguments in Sect. 2, especially the use of the Birkhoff Ergodic Theorem in Proposition 2.3, is taken from [B-S].

The heart of this paper (Lemma 3.2) is a fixed point result that detects a finite invariant set $K$, so that the relative mapping class determined by $f$ and $K$ is pseudo-Anosov. The proof of this lemma is essentially a subset of the proof of Theorem 9.1 in [H1]. The techniques from [H1] can also be used to prove Lemmas 2.1 and 2.2 .

\section{Relative Pseudo-Anosov Homeomorphisms}

We say that $\phi: \mathbf{A} \rightarrow \mathbf{A}$ is pseudo-Anosov relative to a finite invariant set $K$ if it satisfies all of the properties of a pseudo-Anosov homeomorphism (see [T] or $[\mathrm{F}-\mathrm{L}-\mathrm{P}]$ ) except that the associated stable and unstable foliations may have 1-pronged singularities at points in $K$. Equivalently, let $N$ be the compact surface obtained from $\mathbf{A} \backslash K$ by compactifying each puncture with a boundary circle; let $p: N \rightarrow \mathbf{A}$ be the map that collapses these boundary circles to points. Then $\phi$ is pseudo-Anosov relative to $K$, if and only if there is a pseudo-Anosov homeomorphism $\Phi: N \rightarrow N$ such that $\phi p=p \Phi$.

Proposition 1.1. If $\phi: \mathbf{A} \rightarrow \mathbf{A}$ is pseudo-Anosov relative to some finite invariant set $K$, then each $R(\tilde{\phi})$ is a closed interval. For each $r \in R(\widetilde{\phi})$, there is a compact invariant set $Q_{r} \subset \mathbf{A}$ such that $\varrho(\tilde{\phi}, q)=r$ for all $q \in Q_{r}$. Moreover, if $r \in \operatorname{int}(R(\widetilde{\phi}))$, then we may choose $Q_{r} \subset \operatorname{int}(\mathbf{A} \backslash K)$. 
Proof of Proposition 1.1. Let $p: N \rightarrow \mathbf{A}$ and $\Phi: N \rightarrow N$ be as in the definition of a relative pseudo-Anosov homeomorphism. We can compute $\varrho(\tilde{\phi}, x)$ using $\Phi$ instead of $\phi$ as follows. Let $\tilde{K} \subset \tilde{\mathbf{A}}$ be the full pre-image of $K \subset \mathbf{A}$. Let $\tilde{N}$ be the infinite cyclic cover of $N$ obtained from $\tilde{\mathbf{A}} \backslash \widetilde{K}$ by compactifying each puncture with a boundary circle, and let $\tilde{p}: \tilde{N} \rightarrow \tilde{\mathbf{A}}$ be the map that collapses these boundary circles to points. A lift $\tilde{\phi}: \tilde{\mathbf{A}} \rightarrow \tilde{\mathbf{A}}$ of $\phi: \mathbf{A} \rightarrow \mathbf{A}$ determines a lift $\tilde{\Phi}: \tilde{N} \rightarrow \tilde{N}$ of $\Phi: N \rightarrow N$ such that $\tilde{p} \tilde{\Phi}=\tilde{\phi} \tilde{p}$. Choose $y \in p^{-1}(x) \subset N$ and a lift $\tilde{y} \in \tilde{N}$ of $y$; define $\varrho(\tilde{\Phi}, y)=\lim _{n \rightarrow \infty}\left(p_{1} \tilde{p} \tilde{\Phi}^{n}(\tilde{y})\right.$ $\left.-p_{1} \tilde{p} \tilde{y}\right) / n=\lim _{n \rightarrow \infty}\left(p_{1} \tilde{\phi}^{n} \tilde{p}(\tilde{y})-p_{1} \tilde{p} \tilde{y}\right) / n=\varrho(\tilde{\phi}, x)$ if this limit exists.

Choose a Markov partition of $N$ into topological rectangles $\left\{R_{1}, \ldots, R_{n}\right\}$ such that the diameter of each $R_{i}$ and each $\Phi\left(R_{i}\right)$ is less than $1 / 4$ and such that each nonempty $\Phi\left(R_{i}\right) \cap\left(\right.$ int $\left.R_{j}\right)$ is connected. Let $B=\left(b_{i j}\right)$ be the transition matrix for $\Phi: N \rightarrow N$ with respect to this partition; i.e. $b_{i j}=1$ if $\Phi\left(R_{i}\right) \cap\left(\operatorname{int} R_{j}\right) \neq \emptyset$ and $b_{i j}=0$ otherwise. We say that a sequence $S=\left\{s_{i}\right\}$ of integers $1 \leqq s_{i} \leqq n$ is admissible if each $b_{s_{i} s_{i+1}}=1$. Since $B$ is an irreducible matrix (Expose 10 of $[\mathrm{F}-\mathrm{L}-\mathrm{P}]$ ), there is a constant $L$ so that for any $1 \leqq i, j \leqq n$, there is an admissible sequence of length at most $L$ starting at $i$ and ending at $j$.

An admissible sequence $S=\left\{s_{i}: 0 \leqq i \leqq m\right\}$ is a partial address for $y \in N$ if $\Phi^{i}(y) \in R_{s_{i}}$ for $0 \leqq i \leqq m$. We say that $S$ has average rotation greater than $r$ if $\left(p_{1} \tilde{p} \tilde{\Phi}^{m}(\tilde{y})\right.$ $\left.-p_{1} \tilde{p}(\tilde{y})\right) / m>r$ for every lift $\tilde{y} \in \tilde{N}$ of every point $y \in N$ that has $S$ as a partial address. We define average rotation less than $r$ similarly.

Suppose that $\varrho\left(\widetilde{\Phi}, y_{1}\right)=r_{1}<r<r_{2}=\varrho\left(\widetilde{\Phi}, y_{2}\right)$ for some $y_{1}, y_{2} \in N$ and $r, r_{1}, r_{2} \in \mathbf{R}$. Choose $1 \leqq i_{0} \leqq n$ so that $b_{i_{0} 1}=1$. By adding admissible sequences of length at most $L$ to the beginning and end of a long partial address for $y_{1}$, we construct an admissible sequence $U$ that starts with 1 , ends with $i_{0}$ and that has average rotation less than $r$. Similarly we can use $y_{2}$ to construct an admissible sequence $V$ that starts with 1 , ends with $i_{0}$ and that has average rotation greater than $r$.

Define the infinite admissible sequence $S=W_{1} W_{2} W_{3} \ldots$ by $W_{1}=U$, by $W_{i+1}=U$ if $W_{1} W_{2} \ldots W_{i}$ has average displacement greater than $r$ and by $W_{i+1}=V$ otherwise. Choose $y \in N$ so that $\Phi^{i}(y) \in R_{s_{i}}$ for all $i>0$ (Expose 10 of [F-L-P]), and note that $\left|p_{1} \tilde{p} \tilde{\Phi}^{k}(\tilde{y})-p_{1} \tilde{p}(\tilde{y})-k r\right|$ is bounded independently of $k>0$. Let $Q_{r}^{*}$ be the $\omega$-limit set of the $\Phi$-orbit of $y$ and let $Q_{r}=p Q_{r}^{*}$. Then $\varrho\left(\tilde{\Phi}, q^{*}\right)=r$ for all $q^{*} \in Q_{r}^{*}$ and $\varrho(\tilde{\phi}, q)=r$ for all $q \in Q_{r}$.

We have shown that $R(\tilde{\phi})$ is an interval and that each $r \in \operatorname{int}(R(\tilde{\phi}))$ is realized on a compact invariant set. By choosing $y_{1}, y_{2} \in \operatorname{int}(N)$ and by choosing $U$ and $V$ sufficiently long, we guarantee that $Q_{r}^{*} C \operatorname{int}(N)$ and hence that $Q_{r} \subset \operatorname{int}(\mathbf{A} \backslash K)$.

Suppose now that $r$ is the upper endpoint of $R(\widetilde{\phi})$. (The argument for the lower endpoint is similar.) Choose an increasing sequence $r_{l} \rightarrow r$. We claim that there exist constants $M_{l}>0$ so that for any $1 \leqq i, j \leqq n$, there is an admissible sequence $V=V(l, i, j)=\left\{v_{k}: 0 \leqq k \leqq M_{l}\right\}$ such that:

1. $v_{0}=i$,

2. $v_{M_{l}}=j$,

3. $V$ has average rotation greater than $r_{l}$,

4. For all $0<l^{\prime}<l$ and $0 \leqq a \leqq M_{l}-M_{l^{\prime}}$, the admissible sequence $\left\{v_{a}, \ldots, v_{a+M_{l^{\prime}}}\right\}$ has average rotation greater than $r_{l^{\prime}}$.

The construction is inductive. Suppose that we have constructed $V\left(l^{\prime}, i, j\right)$ and $M_{l^{\prime}}$ for all $0<l^{\prime}<l$ and all $1 \leqq i, j \leqq n$. Construct $V^{1}(l, i, j)=\left\{v_{k}^{1}: 0 \leqq k \leqq M_{l}\right\}$ 
satisfying (1)-(3) from a long partial address for some $y_{l+1} \in N$ satisfying $\varrho\left(\widetilde{\Phi}, y_{l+1}\right)$ $=r_{l+1}$, by adding appropriate initial and terminal sequences of length at most $L$. If (4) fails for some $l^{\prime}$ and $a$, replace $\left\{v_{a}^{1}, \ldots, v_{a+M_{l^{\prime}}}^{1}\right\}$ in $V^{1}(l, i, j)$ by $V\left(l^{\prime}, v_{a}^{1}, v_{a+M_{l^{\prime}}}^{1}\right)$ to obtain $V^{2}(l, i, j)$. Then $V^{2}(l, i, j)$ also satisfies (1)-(3) and if $V^{1}(l, i, j)$ is a partial address for $z_{1}$ and $V^{2}(l, i, j)$ is a partial address for $z_{2}$, then $p_{1} \tilde{p} \tilde{\Phi}^{M_{l}}\left(\tilde{z}_{2}\right)-p_{1} \tilde{p}\left(\tilde{z}_{2}\right)$ $>p_{1} \tilde{p} \tilde{\Phi}^{M_{l}}\left(\tilde{z}_{1}\right)-p_{1} \tilde{p}\left(\tilde{z}_{1}\right)+1 / 2$. After finitely many such replacements we arrive at $V(l, i, j)$.

Define $S$ to be the infinite admissible sequence $S=V\left(1,1, i_{0}\right) \quad V\left(2,1, i_{0}\right)$ $V\left(3,1, i_{0}\right) \ldots$ and let $y \in N$ satisfy $\Phi^{i}(y) \in R_{s_{i}}$ for all $i>0$. Define $Q_{r}^{*}$ to be the $\omega$-limit set of the $\Phi$-orbit of $y$ and define $Q_{r}=p Q_{r}^{*}$. Then $\varrho(\tilde{\phi}, q)=r$ for every $q \in Q_{r}$.

A key dynamical property of a pseudo-Anosov homeomorphism is that it has minimal complexity among all elements in its mapping class. The following proposition is another example of this phenomenon.

Proposition 1.2. Suppose that $\phi: \mathbf{A} \rightarrow \mathbf{A}$ is pseudo-Anosov relative to $K$ and that $f: \mathbf{A} \rightarrow \mathbf{A}$ is homotopic to $\phi$ relative to $K$. If $\tilde{f}: \tilde{\mathbf{A}} \rightarrow \tilde{\mathbf{A}}$ and $\tilde{\phi}: \tilde{\mathbf{A}} \rightarrow \tilde{\mathbf{A}}$ are lifts that are equivariantly homotopic $\operatorname{rel} \tilde{K}$, then $R(\tilde{f}) \supset \operatorname{int}(R(\tilde{\phi}))$. Moreover, for each $r \in \operatorname{int}(R(\tilde{\phi}))$, there is a compact $f$-invariant set $Q_{r}^{\prime}$ such that $\varrho\left(\tilde{f}, q^{\prime}\right)=r$ for all $q^{\prime} \in Q_{r}^{\prime}$.

Proof of Proposition 1.2. Fix $r \in \operatorname{int}(R(\tilde{\phi}))$. Let $p: N \rightarrow \mathbf{A}, \Phi: N \rightarrow N, \tilde{p}: \tilde{N} \rightarrow \tilde{\mathbf{A}}$ and $\widetilde{\Phi}: \tilde{N} \rightarrow \tilde{N}$ be as in the proof of Proposition 1.1. Then $\varrho(\tilde{\Phi}, y)=\varrho(\tilde{\phi}, p(y))$ for all $y \in N$ at which these limits are defined. Let $Q_{r}^{*}\left(\operatorname{int}(N)\right.$ and $Q_{r}=p\left(Q_{r}^{*}\right)$ be as in Proposition 1.1.

For all $n>0$, there exist $F_{n}: N \rightarrow N$ and $f_{n}: \mathbf{A} \rightarrow \mathbf{A}$ such that $p F_{n}=f_{n} p$ and such that $f=f_{n}$ on $K$ and on the complement in $\mathbf{A}$ of the $1 / n$-neighborhood of $K$. (Of course, if $f$ is well behaved on a neighborhood of $K$, then there exists $F: N \rightarrow N$ such that $p F=f p$.) Let $\tilde{f}_{n}: \tilde{\mathbf{A}} \rightarrow \tilde{\mathbf{A}}$ be the lift of $f_{n}: A \rightarrow A$ that is equivariantly homotopic, $\operatorname{rel} \tilde{K}$, to $\tilde{f}: \tilde{\mathbf{A}} \rightarrow \tilde{\mathbf{A}}$ and to $\tilde{\phi}: \tilde{\mathbf{A}} \rightarrow \tilde{\mathbf{A}}$; let $\widetilde{F}_{n}: \tilde{N} \rightarrow \tilde{N}$ be the lift of $F_{n}: N \rightarrow N$ that satisfies $\tilde{p} \tilde{F}_{n}=\widetilde{f}_{n} \tilde{p}$. Then $\widetilde{F}_{n}$ is equivariantly homotopic to $\tilde{\Phi}$ and $\varrho\left(\widetilde{F}_{n}, w\right)=\lim _{k \rightarrow \infty}\left(p_{1} \tilde{p} \widetilde{F}_{n}^{k}(\tilde{w})\right.$ $\left.-p_{1} \tilde{p} \tilde{w}\right) / k=\lim _{k \rightarrow \infty}\left(p_{1} \tilde{f}_{n}^{k} \tilde{p}(\tilde{w})-p_{1} \tilde{p} \tilde{w}\right) / k=\varrho\left(\tilde{f}_{n}, p(w)\right)$ for all $w \in N$ on which these limits are defined.

We say that the $\Phi$-orbit of $y$ shadows the $F_{n}$-orbit of $w$ in $\tilde{N}$ if there exist $C>0$ and lifts $\tilde{w}, \tilde{y} \in \tilde{N}$ so that $\widetilde{D}\left(\widetilde{\Phi}^{k}(\tilde{y}), \widetilde{F}_{n}^{k}(\tilde{w})\right)<C$ for all $k \in \mathbf{Z}$, where $\tilde{D}$ is an equivariant metric on $\tilde{N}$. If the $\Phi$-orbit of $y$ shadows the $F_{n}$-orbit of $w$ in $\tilde{N}$, then $\varrho(\tilde{\Phi}, y)$ $=\varrho\left(\tilde{F}_{n}, w\right)$.

Fig. 1

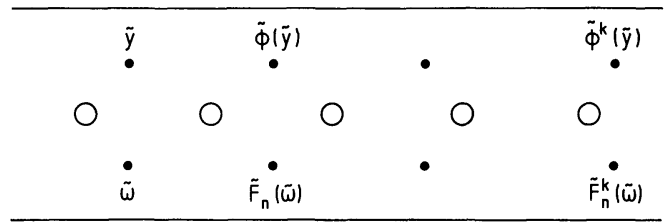

Let $\hat{N}$ be the universal cover of $N$, and let $\hat{\Phi}: \hat{N} \rightarrow \hat{N}$ and $\hat{F}_{n}: \hat{N} \rightarrow \hat{N}$ be equivariantly homotopic lifts of $\Phi: N \rightarrow N$ and $F_{n}: N \rightarrow N$ respectively. We say that the $\Phi$-orbit of $y$ shadows the $F_{n}$-orbit of $w$ in $\hat{N}$ (this is called global shadowing in $[\mathrm{H} 2])$ if there exist $C>0$ and lifts $\hat{w}, \hat{y} \in \hat{N}$ such that $\hat{D}\left(\hat{\Phi}^{k}(\hat{y}), \hat{F}_{n}^{k}(\hat{w})\right)<C$ for all $k \in \mathbf{Z}$, 
where $\hat{D}$ is an equivariant metric on $\hat{N}$. If the $\Phi$-orbit of $y$ shadows the $F_{n}$-orbit of $w$ in $\hat{N}$, then obviously the $\Phi$-orbit of $y$ also shadows the $F_{n}$-orbit of $w$ in $\tilde{N}$. We are therefore reduced to proving the following lemma.

Lemma 1.3. For all $\varepsilon>0$, there exists $\delta>0$, so that if $Q^{*} \subset N$ is a non-empty compact $\phi$-invariant set satisfying $\operatorname{dist}\left(Q^{*}, \partial N\right)>\varepsilon$, then for all $n>0$ there exists a non-empty compact $F_{n}$-invariant set $Q^{\prime *}(n) \subset N$ such that $\operatorname{dist}\left(Q^{\prime *}(n), \partial N\right)>\delta$ and such that for all $w \in Q^{*}(n)$, there exists $y \in Q^{*}$ so that the $\Phi$-orbit of $y$ shadows the $F_{n}$-orbit of $w$ in $\hat{N}$.

Proof of Lemma 1.3. This is a straightforward extension of Theorem 1 of [H2]. The stable and unstable foliations for $\Phi$ determine a function $\hat{D}_{F o l}: \hat{N} \times \hat{N} \rightarrow[0, \infty)$ (as in 1.4 of [H2]) that has all the properties of an equivariant metric except that $\hat{D}_{F o l}\left(\hat{z}_{1}, \hat{z}_{2}\right)=0$ when $\hat{z}_{1}$ and $\hat{z}_{2}$ lie in the same component of the pre-image of $\partial N$. For sufficiently small $\delta_{0}$, the $D_{\mathrm{Fol}}$-diameter of a component of the full pre-image of $N_{\delta_{0}}(\partial N)$ is less than one. In particular, $\widehat{D}_{F o l}\left(\hat{\Phi}(\hat{z}), \widehat{F}_{n}(\hat{z})\right)$ and $\hat{D}_{F o l}\left(\hat{\Phi}^{-1}(\hat{z}), \hat{F}_{n}^{-1}(\hat{z})\right)$ are uniformly bounded independently of $n$ and $\hat{z}$. This implies (cf. the proof of Lemma 2.2 in [H2]) that the constant $C$ used in the definition of shadowing in $\hat{N}$ can be chosen independently of $y, w$, and $n$. This is sufficient (cf. the proof of Theorem 2 in [H2]) to imply the existence of $Q^{\prime *}(n)$ with the appropriate shadowing properties; it remains to show that $\operatorname{dist}\left(Q^{\prime *}(n), \partial N\right)$ has a lower bound that is independent of $n$.

There exists $K>0$ so that if $\operatorname{dist}(y, \partial N)>\varepsilon$, then $\sup _{|k|<K} \hat{D}_{F o l}\left(\hat{\Phi}^{k}(\hat{y}), \hat{A}\right)>C+1$ for every lift $\hat{y}$ of $y$ and component $\hat{A}$ of the pre-image of $\partial N$. Choose $\delta>0$ so that if $w \in N_{\delta}(\partial N)$, then $F_{n}^{k}(w) \in N_{\delta_{0}}(\partial N)$ for all $|k|<K$ and all sufficiently large $n$.

\section{The $\boldsymbol{A} \boldsymbol{A}$ Hypothesis}

Throughout this section $\tilde{f}: \tilde{\mathbf{A}} \rightarrow \tilde{\mathbf{A}}$ is a fixed lift of a fixed homeomorphism $f: \mathbf{A} \rightarrow \mathbf{A}$; we will write $\varrho(x)$ for $\varrho(\widetilde{f}, x)$. Most of the results in this section are contained in [B-S].

Lemma 2.1. Suppose that $\varrho(x)$ is defined and that $Y$ is the $\omega$-limit set of $x$. Then either $\varrho(y)=\varrho(x)$ for all $y \in Y$, or there are periodic orbits in $\mathbf{A}$ with prime period and with rotation numbers arbitrarily close to $\varrho(x)$.

Proof of Lemma 2.1. If the lemma fails, then (cf. Theorem 6.19 of [Wa]) there exists a point $y \in Y$ such that $\varrho(y) \neq \varrho(x)$. For concreteness, assume that $\varrho(y)>\varrho(x)$ and choose $\varrho(y)>p / q>\varrho(x)$. We will show that there exists a periodic point $z \in \mathbf{A}$ such that $\varrho(z)=p / q$.

Let $h=T^{-p} \widetilde{f}^{q}: \tilde{\mathbf{A}} \rightarrow \tilde{\mathbf{A}}$. Then $\lim _{n \rightarrow \infty} p_{1} h^{n}(\tilde{y})=\infty$ and $\lim _{n \rightarrow \infty} p_{1} h^{n}(\tilde{x})=-\infty$ for any lifts $\tilde{y}$ and $\tilde{x}$ of $y$ and $x$. Fix $\varepsilon>0$. For each $i>0$, choose lifts $\tilde{y}_{i}$ of $y$ and $\tilde{x}_{i}$ of $x$ and choose segments $\tilde{Y}_{i}=\left\{h^{j}\left(\tilde{y}_{i}\right): a_{i}<j<b_{i}\right\}$ of the $h$-orbit of $\tilde{y}_{i}$ and $\tilde{X}_{i}=\left\{h^{j}\left(\tilde{x}_{i}\right): c_{i}<j<d_{i}\right\}$ of the $h$-orbit of $\tilde{x}_{i}$ such that

1. $p_{1} h^{a_{i}}\left(\tilde{y}_{i}\right)<-10^{i}$

2. $p_{1} h^{b_{i}}\left(\tilde{y}_{i}\right)>10^{i}$,

3. $\operatorname{dist}\left(h^{b_{i}}\left(\tilde{y}_{i}\right), h^{c_{i}}\left(\tilde{x}_{i}\right)\right)<\varepsilon$,

4. $\operatorname{dist}\left(h^{a_{i+1}}\left(\tilde{y}_{i+1}\right), h^{d_{i}}\left(\tilde{x}_{i}\right)\right)<\varepsilon$. 
The $\varepsilon$-pseudo-orbit $\widetilde{Y}_{1} \widetilde{X}_{1} \widetilde{Y}_{2} \tilde{X}_{2} \ldots$ has accumulation points, so by [Fr], $h$ has a fixed point $\tilde{z}$. Let $z \in \mathbf{A}$ be the projected image of $\tilde{z}$.

Lemma 2.2. Suppose that $Y_{i}$ is an $\omega$-limit set, that $\varrho \mid Y_{i}=r_{i} \rightarrow r$, and that $Y_{i} \rightarrow Y$ in the Hausdorf topology on closed sets. Then either $\varrho \mid Y=r$ or there exist periodic orbits in A with prime periods and with rotation numbers arbitrarily close to $r$.

Proof of Lemma 2.2. The proof is similar to that of Lemma 2.1 and is left to the reader.

We say that $r$ satisfies the $p A$-hypothesis if there are periodic orbits $X_{i}$, a closed invariant set $X$, an invariant measure $\mu$ with support in $X$, a (not necessarily invariant) set $B \subset X$ of positive $\mu$-measure and $\varepsilon>0$ such that

(pA-i) $X_{i} \rightarrow X$ in the Hausdorf topology.

(pA-ii) Either $\varrho\left(X_{i}\right)<r<r+\varepsilon<\varrho(b)$ for all $b \in B$ and all sufficiently large $i$ or $\varrho\left(X_{i}\right)>r>r-\varepsilon>\varrho(b)$ for all $b \in B$ and all sufficiently large $i$.

This definition is motivated by Lemma 3.1 and the following proposition.

Proposition 2.3. For all $r$ in the closure of $R(\tilde{f})$, one of the following holds:

1. There is a compact invariant set $Q$ such that $\varrho(q)=r$ for all $q \in Q$.

2. There is an f-invariant measure $\mu$ such that $\varrho(x)=r$ for $\mu$-a.e. $x \in \mathbf{A}$.

3. $r$ satisfies the pA-hypothesis.

Moreover, the set of values of $r$ that satisfy (2), but not (1) or (3), is discrete.

Proof of Proposition 2.3. Lemmas 2.1 and 2.2 imply that either (1) holds or there exist periodic orbits $X_{i}$ with $\varrho\left(X_{i}\right)=r_{i} \rightarrow r$. After passing to a subsequence, we may assume that $\left\{X_{i}\right\}$ converges in the Hausdorf topology to a closed invariant set $X$ and that the unique invariant measure $\mu_{i}$ on $X_{i}$ converges in the weak-star topology to an invariant measure $\mu$ with support in $X$.

Let $g: \mathbf{A} \rightarrow \mathbf{A}$ be the displacement function $g(z)=p_{1} \tilde{f}(\tilde{z})-p_{1}(\tilde{z})$, where $\tilde{z} \in \tilde{\mathbf{A}}$ is any lift of $z \in \mathbf{A}$. Then $\varrho(z)=\lim _{n \rightarrow \infty}(1 / n)\left(\sum_{k=0}^{n-1} g\left(f^{k}(z)\right)\right)$ and the Birkhoff Ergodic Theorem implies that $\int_{X_{i}} g d \mu_{i}=\int_{X_{i}} \varrho d \mu_{i}=r_{i}$ and that $\int_{X} g d \mu=\int_{X} \varrho d \mu$. Since $\mu_{i} \rightarrow \mu$ in the weak-star topology, $\int_{X_{i}} g d \mu_{i} \rightarrow \int_{X} g d \mu$. We conclude that $\int_{X} \varrho d \mu=r$. Either $\varrho(x)=r \mu$-a.e. or $r$ satisfies the $p A$-hypothesis.

It remains to show that the set $D$ of points in the closure of $R(\tilde{f})$ that satisfy (2) but not (1) or (3) is discrete. Suppose that $r_{i} \in D$ and that $r_{i} \rightarrow r$. We will show that $r \notin D$. Lemmas 2.1 and 2.2 imply that there are periodic orbits $Y_{i}$ such that $\varrho\left(Y_{i}\right) \rightarrow r$. We may assume without loss that $\left\{Y_{i}\right\}$ converges in the Hausdorf topology to a closed invariant set $Y$. If $\varrho \mid Y=r$ we are done. We may therefore assume that there is an invariant measure $v$ with support in $Y$ such that $\int_{Y} \varrho d v \neq r$ (cf. Theorem 6.19 of [Wa]). Suppose for concreteness that each $r_{i}<r$; the case $r_{i}>r$ is similar. If $\int_{Y} \varrho d v<r$, then $r_{i}$ would satisfy the $p A$-hypothesis for all sufficiently large $i$. Thus $\int_{Y} \varrho d v>r$ and $r$ satisfies the $p A$-hypothesis. 


\section{Proof of Theorem 0.1}

As in Sect. 2, $\tilde{f}: \tilde{\mathbf{A}} \rightarrow \tilde{\mathbf{A}}$ is a fixed lift of a fixed homeomorphism $f: \mathbf{A} \rightarrow \mathbf{A}$ and we write $\varrho(x)$ for $\varrho(\widetilde{f}, x)$. The results in Sects. 1 and 2 reduce Theorem 0.1 to the following proposition.

Proposition 3.1. If $r$ satisfies the $p A$-hypothesis, then there exists $n>0$, an $f^{n}$ invariant finite set $K$ and a homeomorphism $\phi: \mathbf{A} \rightarrow \mathbf{A}$ such that:

1. $\phi$ is pseudo-Anosov relative to $K$,

2. $\phi \simeq f^{n} \operatorname{rel} K$,

3. $n r \in \operatorname{int}(R(\tilde{\phi}))$, where $\tilde{\phi}: \tilde{\mathbf{A}} \rightarrow \tilde{\mathbf{A}}$ is the lift of $\phi$ that is equivariantly homotopic to $\tilde{f}^{n} \operatorname{rel} \tilde{K}$.

The following lemma is the heart of this paper. Its proof is postponed until after the proof of Proposition 3.1.

Lemma 3.2. Suppose that $r$ satisfies the $p A$-hypothesis. Then there exist $f$-periodic orbits $Y_{1}$ and $Y_{2}$ that have distinct prime periods and that satisfy the following property: for any homeomorphism $g: \mathbf{A} \rightarrow \mathbf{A}$ that is homotopic to $f$ relative to $Y_{1} \cup Y_{2}$, there is a g-periodic orbit $Z$ such that either $\varrho(Z)<r<\varrho\left(Y_{1}\right), \varrho\left(Y_{2}\right)$ or $\varrho\left(Y_{1}\right), \varrho\left(Y_{2}\right)$ $<r<\varrho(Z)$.

Proof of Proposition 3.1. Let $Y_{1}$ and $Y_{2}$ be as in Lemma 3.2, let $N$ be the compact surface obtained from $\mathbf{A} \backslash\left(Y_{1} \cup Y_{2}\right)$ by compactifying each end by a circle and let $p: N \rightarrow \mathbf{A}$ be the quotient map.

The restriction $f \mid\left(\mathbf{A} \backslash\left(Y_{1} \cup Y_{2}\right)\right)$ determines an element $\mu$ of the mapping class group of $N$. If $\mu$ is a pseudo-Anosov class, define $n=1, K=Y_{1} \cup Y_{2}$ and $\phi=p \Phi p^{-1}$, where $\Phi$ is a pseudo-Anosov representative of $\mu$. Properties (1) and (2) are immediate. Property (3) follows from Lemma 1.1.

If $\mu$ were a finite order class, then it would have a representative $G: N \rightarrow N$ whose projected image $g=p G p^{-1}: \mathbf{A} \rightarrow \mathbf{A}$ was conjugate to a rigid rotation (see [H3], for example). Since $Y_{1}$ and $Y_{2}$ have distinct periods, this is impossible.

We may therefore assume that $\mu$ is reducible [T]. Choose a reducing set $\Gamma$ for $\mu$; i.e. a disjoint union $\Gamma=\left\{\gamma_{1}, \ldots, \gamma_{k}\right\}$ of non-peripheral, non-parallel, simple closed curves in $N$ that is setwise preserved by a representative $G: N \rightarrow N$ of $\mu$.

We first observe that for each $1 \leqq j \leqq k, G$ setwise fixes $\gamma_{j}$. If $\gamma_{j}$ separates the components of $\partial \mathbf{A}$ then this is immediate. If $\gamma_{j}$ bounds a disk $D_{j}$ in $\mathbf{A}$, let $l_{j}$ be the smallest positive integer such that $G^{l_{j}}\left(\gamma_{j}\right)=\gamma_{j}$. If $Y_{i} \cap D_{j} \neq \emptyset$, then the period of $Y_{i}$ is the product of $l_{j}$ with the cardinality of $Y_{i} \cap D_{j}$. The claim now follows from the fact that the periods of $Y_{i}$ are distinct primes.

We next observe that each $\gamma_{j}$ separates $Y_{1}$ from $Y_{2}$. If $\gamma_{j}$ separates the components of $\partial \mathbf{A}$, then this follows from the fact that $\gamma_{j}$ is non-peripheral. If $\gamma_{j}$ bounds a disk $D_{j}$, then $D_{j}$ cannot intersect both $Y_{1}$ and $Y_{2}$ since these orbits have distinct rotation numbers. By our first observation, $D_{j}$ contains $Y_{i}$ if it intersect $Y_{i}$. Since $\gamma_{j}$ is not null-homotopic in $N, \gamma_{k}$ separates $Y_{1}$ from $Y_{2}$.

The five possible configurations for $\Gamma$ are shown below; the components of $\mathbf{A} \backslash \Gamma$ whose union contains $Y_{1} \cup Y_{2}$ are labelled $A_{1}$ and $A_{2}$.

Let $P$ be the union of small product neighborhoods of the $\gamma_{j}$ 's. Let $N_{i}$ be the component of $N \backslash P$ corresponding to $A_{i}$. We may assume that $\Gamma$ is a maximal 
reducing set and hence that the element of the mapping class group of $N_{i}$ determined by $G \mid N_{i}$ is irreducible. Let $H_{i}: N_{i} \rightarrow N_{i}$ be a representative of this class that is either pseudo-Anosov or finite order. Then $\cup H_{i}: \cup N_{i} \rightarrow \cup N_{i}$ extends to a homeomorphism $H: N \rightarrow N$ whose nonwandering set is contained in $\cup N_{i}$. Let $\widetilde{H}: \widetilde{N} \rightarrow \widetilde{N}$ be the lift of $H: N \rightarrow N$ determined by $\widetilde{f}: \widetilde{\mathbf{A}} \rightarrow \tilde{\mathbf{A}}$. Denote $\left\{\varrho(\widetilde{H}, x): x \in N_{i}\right\}$ by $R\left(H_{i}\right)$.

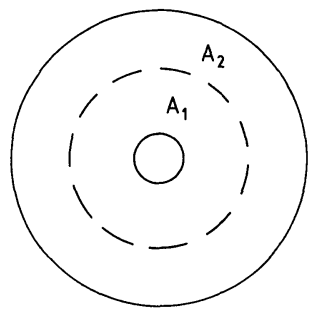

(a)

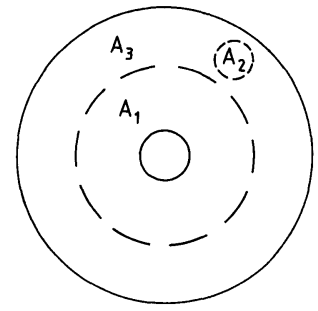

(b)

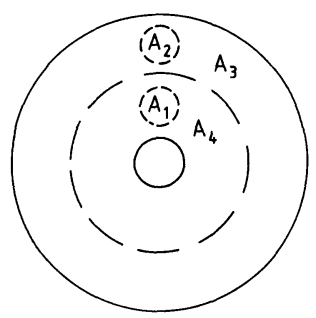

(c)

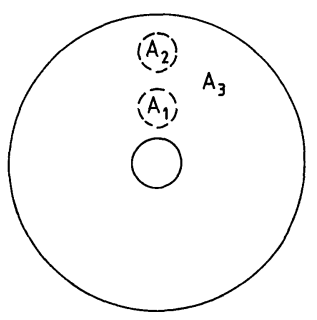

(d)

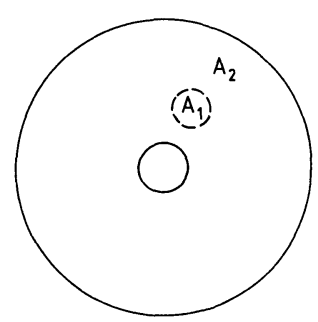

(e)

Fig. 2

We consider the five possibilities. In Figure (3.1-a), $A_{1}$ and $A_{2}$ are annuli and $R(\tilde{H})=R\left(\tilde{H}_{1}\right) \cup R\left(\tilde{H}_{2}\right)$. If $H_{i}$ is finite order, then $R\left(\tilde{H}_{i}\right)=\varrho\left(Y_{i}\right)$. Thus either $H_{1}$ or $H_{2}$, say $H_{1}$, is pseudo-Anosov and $r \in \operatorname{int}\left(R\left(\tilde{H}_{1}\right)\right)$. Define $n=1, K=Y_{1}$ and $\phi=s p H_{1} p^{-1} s^{-1}$, where $s: \mathbf{A}_{1} \rightarrow \mathbf{A}$ is a homeomorphism that is homotopic to the identity relative to $Y_{1}$.

In Figure (3.1-b), $A_{1}$ is an annulus, $A_{2}$ is a disk and $N_{3}$ is a three times punctured sphere. Since $H_{3}: N_{3} \rightarrow N_{3}$ setwise preserves the components of $\partial N_{3}$, $H_{3}=$ identity. Moreover, $\varrho(\tilde{H}, z)=\varrho\left(Y_{2}\right)$ for all $z \in N_{2}$. Thus $R(\tilde{H})=R\left(\widetilde{H}_{1}\right) \cup \varrho\left(Y_{2}\right)$ and the proof concludes as in the previous case.

In Figure (3.3-c), $A_{1}$ and $A_{2}$ are disks while $N_{3}$ and $N_{4}$ are three punctured spheres. Thus $R(H)=\varrho\left(Y_{1}\right) \cup \varrho\left(Y_{2}\right)$ which is impossible. 
In Figure (3.3-d), $A_{1}$ and $A_{2}$ are disks, $N_{3}$ is a four times punctured sphere and $R(\tilde{H})=R\left(\tilde{H}_{3}\right) \cup \varrho\left(Y_{1}\right) \cup \varrho\left(Y_{2}\right)$. As above, $H_{3}$ is pseudo-Anosov and $r \in \operatorname{int}\left(R\left(\tilde{H}_{3}\right)\right)$. Let $n$ be a multiple of the periods of $Y_{1}$ and $Y_{2}$ and let $y_{i} \in Y_{1}, i=1,2$. Let $N^{\prime}$ be the surface obtained from $\mathbf{A} \backslash\left\{y_{1}, y_{2}\right\}$ by compactifying each end with a circle, let $p^{\prime}: N^{\prime} \rightarrow \mathbf{A}$ be the quotient map and let $\mu^{\prime}$ be the element of the mapping class group of $N^{\prime}$ determined by $f^{n} \mid\left(\mathbf{A} \backslash\left\{y_{1}, y_{2}\right\}\right)$. There is a homeomorphism $s: N_{3} \rightarrow N^{\prime}$ so that $s H_{3}^{n} s^{-1}$ is a representative of $\mu^{\prime}$. Define $K=\left\{y_{1}, y_{2}\right\}$ and $\phi=p^{\prime} s H_{3}^{n} s^{-1}\left(p^{\prime}\right)^{-1}$.

In Figure (3.3-e), $A_{1}$ is a disk and $R(\tilde{H})=R\left(\widetilde{H}_{2}\right)+\varrho\left(Y_{1}\right)$. The proof concludes as in the previous case with $K=Y_{2} \cup\left\{y_{1}\right\}$.

The proof of Lemma 3.2 relies on the "homotopy Brouwer Theory" developed in [H1]; see in particular the proofs of Theorem 9.1 and Theorem 4.4 in [H1].

Proof of Lemma 3.2. Let $\left(X_{i}, X, \mu, B, \varepsilon\right)$ be as in the definition of the $p A$-hypothesis. For concreteness, we assume that each $\varrho\left(X_{i}\right)<r$; the case $\varrho\left(X_{i}\right)>r$ is analogous. Choose $r<p / q<r+\varepsilon$ and let $h=T^{-p} \tilde{f}^{q}: \tilde{\mathbf{A}} \rightarrow \tilde{\mathbf{A}}$.

Lemma 3.3. For all $D>0$, there exist $\delta>0, \tilde{y}_{1}, \tilde{y}_{2} \in \tilde{\mathbf{A}}$ and $m, n>0$ so that:

(i) $\tilde{y}_{j}$ covers a point in some $Y_{j}=X_{i_{j}}, j=1,2$,

(ii) $\operatorname{dist}\left(\tilde{y}_{1}, \tilde{y}_{2}\right)<\delta ; \operatorname{dist}\left(h^{m}\left(\tilde{y}_{1}\right), h^{-n}\left(\tilde{y}_{2}\right)\right)<\delta$,

(iii) $h\left(N_{\delta}\left(\tilde{y}_{1}\right)\right) \cap N_{\delta}\left(\tilde{y}_{1}\right)=\emptyset ; h\left(N_{\delta}\left(h^{m}\left(\tilde{y}_{1}\right)\right)\right) \cap N_{\delta}\left(h^{m}\left(\tilde{y}_{1}\right)\right)=\emptyset$,

(iv) $\operatorname{dist}\left(\tilde{y}_{1}, h^{m}\left(\tilde{y}_{1}\right)\right)>D$,

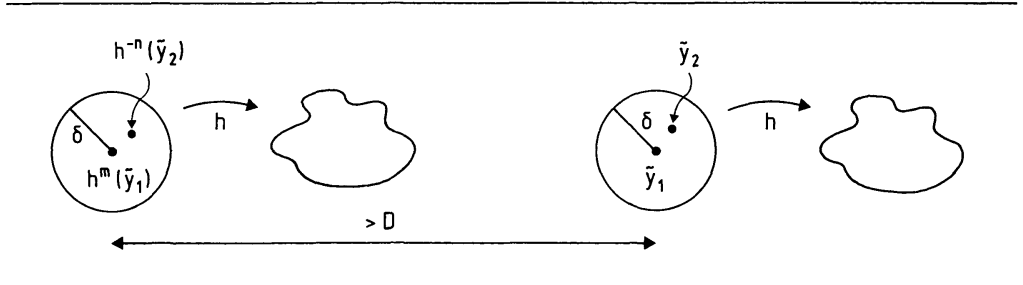

Fig. 3

Proof of Lemma 3.3. Let $\tilde{B} \subset \tilde{X} \subset \tilde{\mathbf{A}}$ be the full pre-images of $B \subset X \subset \mathbf{A}$ and let $\tilde{\mu}$ be the measure on $\tilde{X}$ determined by $\mu$. Since $\varrho \mid B>p / q$, we may assume, after restricting to a subset of $B$ if necessary, that there are constants $D^{\prime}>D>0$ and $\delta>0$ such that:

1. $p_{1} h^{i}(\tilde{b})>p_{1}(\tilde{b})-D^{\prime}$ for all $i>0$ and $\tilde{b} \in \widetilde{B}$,

2. $\operatorname{dist}(h(z), z)<D^{\prime} / 3$ for all $z \in \tilde{\mathbf{A}}$,

3. $h^{i}\left(N_{2 \delta}(\tilde{b}) \cap \widetilde{B}\right) \cap N_{2 \delta}(\widetilde{b})=\emptyset$ for all $i>0$ and $\tilde{b} \in \widetilde{B}$,

4. $h\left(N_{2 \delta}(\widetilde{b})\right) \cap N_{2 \delta}(\widetilde{b})=\emptyset$ for all $\tilde{b} \in \widetilde{B}$.

Fig. 4

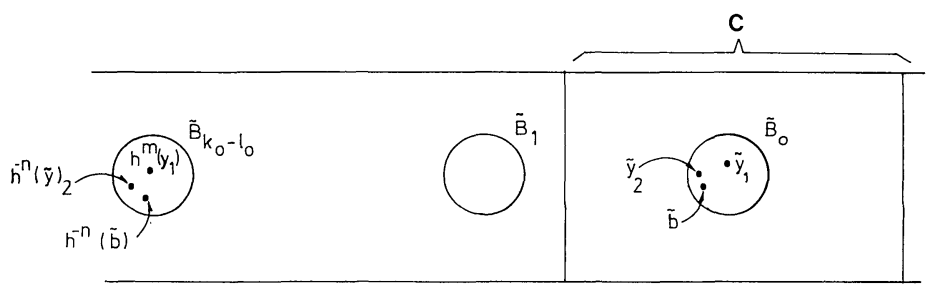


Choose $\tilde{b}_{0} \in \widetilde{B}$ so that $\tilde{\mu}\left(N_{\delta / 2}\left(\widetilde{b_{0}}\right) \cap \widetilde{B}\right)>0$. Since $B$ is contained in the Hausdorf limit of the $X_{i}$ 's, there exists $Y_{1}=X_{i_{1}}, y_{1} \in Y_{1}$, and a lift $\tilde{y}_{1}$ of $y_{1}$ such that $\operatorname{dist}\left(\tilde{y}_{1}, \tilde{b}_{0}\right)<\delta / 2$. If a is a positive multiple of the period of $y_{1}$, then $h^{a}\left(\tilde{y}_{1}\right)=T^{-d}\left(\tilde{y}_{1}\right)$ for some $d>0$. By choosing a to be sufficiently large, we may assume that $d>D^{\prime}$. Let $\widetilde{B}_{0}=N_{\delta}\left(\tilde{y}_{1}\right) \cap \widetilde{B}$ and let $\widetilde{B}_{l}=T^{-l d}\left(\widetilde{B}_{0}\right)=N_{\delta}\left(T^{-l d} \tilde{y}_{1}\right) \cap \widetilde{B}=N_{\delta}\left(h^{l a} \tilde{y}_{1}\right) \cap \widetilde{B}$. Each $B_{l}$ has the same $\tilde{\mu}$ measure, say $c>0$. Properties (2) and (3) imply that the forward orbit of $\widetilde{B}_{l}$ intersects $C=p_{1}^{-1}\left(\left[p_{1}\left(\tilde{y}_{1}\right)-D^{\prime}, p_{1}\left(\tilde{y}_{1}\right)+D^{\prime}\right]\right)$ in a set of $\tilde{\mu}$ measure at least $c$. Since $\tilde{\mu}(C)$ is finite, the forward orbits of the $\widetilde{B}_{l}$ 's can not all be disjoint. By property (1), the forward orbit of $\widetilde{B}_{l}$ is disjoint from $\widetilde{B}_{k}$ for all $k>l$. Thus the forward orbit of $\widetilde{B}_{k_{0}}$ intersects $\widetilde{B}_{l_{0}}$ for some $k_{0}>l_{0}$. Since $h$ commutes with $T$, the forward orbit of $\widetilde{B}_{k_{0}-l_{0}}$ intersects $\widetilde{B}_{0}$. Choose $\tilde{b} \in \widetilde{B}_{0}$ and $n>0$ so that $h^{-n}(\widetilde{b}) \in \widetilde{B}_{k_{0}-l_{0}}$. Let $m=\left(k_{0}-l_{0}\right) a$. Then $\operatorname{dist}\left(\tilde{b}, \tilde{y}_{1}\right)<\delta$, dist $\left(h^{-n}(\tilde{b}), h^{m}\left(\tilde{y}_{1}\right)\right)<\delta$ and $\operatorname{dist}\left(\tilde{y}_{1}, h^{m}\left(\tilde{y}_{1}\right)\right)>D$. Since $\tilde{b}$ is contained in the Hausdorf limit of the $X_{i}{ }^{\prime}$ s, there exists $Y_{2}=X_{i_{2}}, y_{2} \in Y_{2}$ and a lift $\tilde{y}_{2}$ of $y_{2}$ that is so close to $\tilde{b}$, that $\operatorname{dist}\left(\tilde{y}_{2}, \tilde{y}_{1}\right)<\delta$ and $\operatorname{dist}\left(\tilde{h}^{-n}\left(\tilde{y}_{2}\right)\right.$, $\left.\tilde{h}^{m}\left(\tilde{y}_{1}\right)\right)<\delta$.

Let $R=\sup \{\operatorname{dist}(h(z), z): z \in \tilde{\mathbf{A}}\}$ and apply Lemma 3.3 with $D=5 R$. Denote the $h$-orbits of $\tilde{y}_{1}$ and $\tilde{y}_{2}$ by $\mathcal{O}_{1}$ and $\mathcal{O}_{2}$ respectively. Suppose that $g: \mathbf{A} \rightarrow \mathbf{A}$ is homotopic to $f$ relative to $\left(Y_{1} \cup Y_{2}\right)$, and that $h^{\prime}: \tilde{\mathbf{A}} \rightarrow \tilde{\mathbf{A}}$ is the lift of $g^{q}$ that is equivariantly homotopic to $h$ relative to $\left(\mathcal{O}_{1} \cup \mathcal{O}_{2}\right)$. Since fixed points of $h^{\prime}$ correspond to periodic points of $g$ with rotation number $p / q$, it suffices to show that $\operatorname{Fix}\left(h^{\prime} \mid(\operatorname{int}(\tilde{\mathbf{A}})) \neq \emptyset\right.$. We will assume that $\operatorname{Fix}\left(h^{\prime} \mid(\operatorname{int}(\tilde{\mathbf{A}}))=\emptyset\right.$ and arrive at a contradiction.

An $\operatorname{arc} \alpha \operatorname{Cint}(\tilde{\mathbf{A}})$ connecting $x$ to $h(x)$ is called a homotopy translation arc for $x$ with respect to $\left(h, \mathcal{O}_{1} \cup \mathcal{O}_{2}\right)$ if there is a homotopy $F_{t}: \operatorname{int}(\tilde{\mathbf{A}}) \rightarrow \operatorname{int}(\tilde{\mathbf{A}})$ such that:

1. $F_{0}=$ identity,

2. $F_{t} \mid(\mathcal{O}(x) \cup \mathcal{O}(y))=$ identity, $t \in[0,1]$,

3. $F_{1}(h(\alpha)) \cap \alpha=h(x)$.

Note that if $h^{\prime}: \operatorname{int}(\tilde{\mathbf{A}}) \rightarrow \operatorname{int}(\tilde{\mathbf{A}})$ is homotopic to $h$ relative to $\mathcal{O}_{1} \cup \mathcal{O}_{2}$, and if $\alpha$ is a homotopy translation arc with respect to $\left(h, \mathcal{O}_{1} \cup \mathcal{O}_{2}\right)$, then $\alpha$ is also a homotopy translation arc with respect to $\left(h^{\prime}, \mathcal{O}_{1} \cup \mathcal{O}_{2}\right)$.

Let $\tau \subset N_{\delta}\left(\tilde{y}_{1}\right)$ be an arc connecting $\tilde{y}_{1}$ to $\tilde{y}_{2}$. Let $\varrho \subset N_{R}\left(\tilde{y}_{1}\right)$ be an arc connecting $\tilde{y}_{1}$ to $h\left(\tilde{y}_{1}\right)$. Then $h^{-1}(\varrho) \cup \varrho \cup h(\varrho) \subset N_{2 R}\left(\tilde{y}_{1}\right)$ and $h(\tau) \cap \tau=\emptyset$. It follows (cf. Corollary $4.5(\mathrm{c})$ of [H1]) that there is an $\operatorname{arc} \gamma \subset N_{2 R}\left(\tilde{y}_{1}\right)$ that contains $\tilde{y}_{1} \cup \tilde{y}_{2}$ and that is a homotopy translation arc for $\tilde{y}_{1}$ with respect to $\left(h, \mathcal{O}_{1} \cup \mathcal{O}_{2}\right)$. Similarly there are arcs $\alpha \subset N_{2 R}\left(h^{m}\left(\tilde{y}_{1}\right)\right)$ and $\beta \subset N_{2 R}\left(h^{-n}\left(\tilde{y}_{2}\right)\right)$ that contain $\tilde{h}^{m}\left(\tilde{y}_{1}\right) \cup h^{-n}\left(\tilde{y}_{2}\right)$ and that are homotopy translation arcs with respect to $\left(h, \mathcal{O}_{1} \cup \mathcal{O}_{2}\right)$ for $h^{m}\left(\tilde{y}_{1}\right)$ and $h^{-n}\left(\tilde{y}_{2}\right)$ respectively. Note that $\alpha \cap \beta \neq \emptyset$ and that $(\alpha \cup \beta) \cap \gamma=\emptyset$. This contradicts Corollary $4.5(\mathrm{a})$ of $[\mathrm{H} 1]$.

Acknowledgement. I am grateful to Dick Hall for bringing this problem to my attention through his problem list [Hall].

\section{References}

[Au-L] Aubry, S., Le Daeron, P.Y.: The discrete Frenkel-Kontorova model and its extensions. Physica 8d, 381-422 (1983)

[B-S] Barge, M., Swanson, R.: Rotation shadowing properties of circle and annulus maps. Erg. Th. Dyn. Syst. (to appear) 
[F-L-P] Fathi, A., Laudenbach, F., Poenaru, V.: Travaux de Thurston sur les surfaces. Asterisque 66-67 (1979)

[Hall] Hall, G.R.: Some problems on dynamics of annulus maps (preprint)

[H1] Handel, M.: Zero entropy surface diffeomorphisms (preprint)

[H2] Handel, M.: Global shadowing of pseudo-Anosov homeomorphisms. Erg. Th. Dyn. Syst. 5, 173-191 (1985)

[M] Mather, J.: Existence of quasi-periodic orbits for twist homeomorphisms of the annulus. Topology 21, 457-467 (1982)

[T] Thurston, W.P.: On the geometry and dynamics of diffeomorphisms of surfaces. Bull. Am. Math. Soc. 19, 417-431 (1988)

[Wa] Walters, P.: An introduction to ergodic theory. Graduate Texts in Mathematics, vol. 79. Berlin, Heidelberg, New York: Springer 1982

Communicated by J. N. Mather

Received March 9, 1989 
